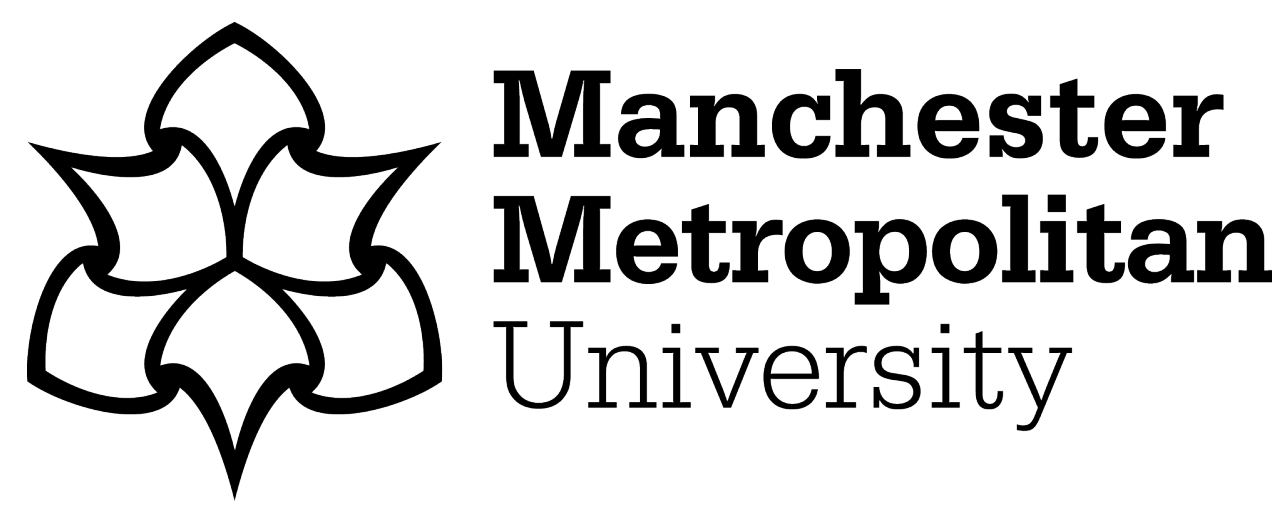

Joseph, Lowdon, Eersels, Kasper, Rogosic, Renato, Heidt, Benjamin, Dilien, Hanne, Steen Redeker, Erik, Peeters, Marloes, van Grinsven, Bart and Cleij, Thomas (2019) Substrate displacement colorimetry for the detection of diarylethylamines. Sensors and Actuators B: Chemical, 282. pp. 137-144. ISSN 0925-4005

Downloaded from: https://e-space.mmu.ac.uk/621849/

Version: Accepted Version

Publisher: Elsevier

DOI: https://doi.org/10.1016/j.snb.2018.11.053

Usage rights: Creative Commons: Attribution-Noncommercial-No Derivative Works 4.0

Please cite the published version 


\title{
Substrate Displacement Colorimetry for the Detection of Diarylethylamines
}

Joseph W. Lowdon ${ }^{1}$, Kasper Eersels ${ }^{1 *}$, Renato Rogosic ${ }^{1}$, Benjamin Heidt ${ }^{1}$, Hanne Diliën ${ }^{1}$, Erik Steen Redeker ${ }^{1}$, Marloes Peeters ${ }^{2}$, Bart van Grinsven ${ }^{1}$, Thomas J. Cleij ${ }^{1}$

1. Maastricht Science Programme, Maastricht University, PO Box 616, 6200 MD, Maastricht, the Netherlands

2. Manchester Metropolitan University, Faculty of Science and Engineering, School of Science and the Environment, Division of Chemistry and Environmental Science, Chester Street, Manchester M1 5GD, United Kingdom.

* Corresponding author: kasper.eersels@maastrichtuniversity.nl

\begin{abstract}
In this work, a novel detection assay for the new psychoactive substance (NPS) 2-methoxiphenidine (2MXP) and other diarylethylamines is introduced. The assay is based on the competitive displacement of dye molecules from molecularly imprinted polymers (MIPs) by the target molecule. The assay was fully characterized by studying the affinity of the MIP for six common dyes, expressed as the binding factor (BF). The results of this study indicate that the mathematical relationship between the BF of a dye and the imprinting factor (IF) for the target could be used for the prediction of the efficacy of the displacement assay. Dye-loaded MIP particles where incubated with the target, two adulterants and two legal pharmacological compounds. The target has a higher affinity for the MIP than the dye and displaces it out of the nanocavities of the receptor leading to a colour change in the filtrate that can be observed with the naked eye. Incubation of the MIP particles with the adulterants and legal medicines did not result in any observable change in absorbance. The robust, fast and low-cost nature of the assay, combined with its tailorable selectivity and generic nature, illustrate its potential as a pre-screening tool for the identification of narcotic substances in unidentified powders.
\end{abstract}

Keywords: Molecularly imprinted polymer, displacement assay, colorimetry, new psychoactive substance identification 


\section{Introduction}

Molecularly imprinted polymers (MIPs) have emerged as synthetic receptors in a wide range of applications over the past few decades $[1,2]$. MIP synthesis is based on cross-linking a polymer in the presence of a template, upon removal of the template nanocavities are created in the polymer network that serve as binding pockets for substrate rebinding [3, 4]. MIPs are able to withstand harsh physical and chemical conditions but display similar binding affinities for their target upon optimizing the synthesis process [57]. Along with their generic, low-cost synthesis process, this makes MIPs interesting for incorporation into biomimetic sensing devices for the detection of a wide variety of compounds in biological samples [8-12]. MIPs have advanced much over years, evolving from bulk polymerized monoliths to more elegant nanoparticles that form the basis of many biosensing devices [13-16].

The replacement of biological receptors by MIPs in alternatives to classical competitive assays in biomedicine was introduced by Vlatakis et al. with the advent of so-called molecular imprinting sorbent assays (MIAs) [17]. Traditionally, these assays combined bulk polymerized MIPs with radio- or fluorescent labeled analytes or enzyme-linked substrates in a competitive binding assay, studied by spectroscopic methods [18-22]. Incubation of pre-loaded MIPs with the target, resulted in displacement of the labeledsubstrates which could be assessed by fluorescent microscopy. The high selectivity and sensitivity of these assays makes them extremely suitable for the detection of trace amounts of analyte but many applications do not require these extreme degrees of sensitivity and selectivity. In this light, several displacement assays were developed that were based on the displacement of a dye that could be observed by absorption spectroscopy, omitting the need for an expensive and labour-intensive procedure such as fluorescent labelling [23-25].

In this study, a novel drug detection assay, coined substrate displacement colorimetry (SDC), is introduced. The assay is able to detect several narcotic compounds based on the colorimetric detection of displaced dye molecules. MIPs were synthesized for the new psychoactive substance (NPS), methoxphenidine (2-MXP). Low-cost drug assays for NPS are highly relevant as most current low-cost field colour test suffer from 
false positives when analysing samples containing adulterants or legal pharmacological compounds and the use of MIPs as synthetic receptors in this assay could overcome this problem to a large extent [26]. Moreover, the production and distribution of NPS have expanded tremendously in recent years to avoid legal legislation. Although these compounds are often considered to be legal highs, yet they are every inch as dangerous and addictive as their illicit counterparts [27-29]. Fast, reliable drug test would help authorities to enforce legislation and provide adequate treatment upon timely intervention.

The classical bulk imprinting approach was chosen due to its easy and scalable synthesis procedure. The synthesis protocol was optimized and binding of six commonly used dyes to the MIP was analysed to determine the binding factor (BF) for each dye. This BF was compared to the MIP imprinting factor (IF) to establish a straightforward prediction model for assessing dye-target combinations. Next, MIPs were loaded with malachite green and washed thoroughly to ensure complete removal of any unbound or weakly bound dye molecules. When exposing these dye-loaded MIPs to a sample containing their target, the dye will be displaced due to the higher affinity the MIP has towards the target analyte, releasing the dye into the surrounding medium (Fig. 1). The assay does not display any cross-selectivity between 2-MXP, two common adulterants and two legal pharmacological compounds. At the same time, the assay is able to detect other diarylethylamines in addition to 2-MXP. In this way, the experiments summarized in this paper illustrate a proof-of-concept for a low cost drug test that allows the end-user to visually determine the presence of narcotics in an unidentified powder and selectively identify e.g. an NPS in a complex mixture, a task that proves hard for routinely used, low-cost drug tests. In addition, a simple absorbance measurement on a spectrophotometer suffices to quantify even tiny amounts of 2-MXP that do not yield a colour reaction that is visible with the bare eye. Finally, a first proof-of-application is provided for a low-cost drug kit by immobilizing the dye-loaded particles directly in a syringe filter. Powder mixtures were dissolved in water and pressed through the filter, making it possible to use the colour of the filtrate as an indicator for the presence of diarylethylamines.

\section{Materials and methods}




\subsection{Chemicals and reagents}

Prior to polymerization, stabilizers were removed from the functional and crosslinking monomers by passing the solutions over a column packed with alumina. All chemicals and solvents were obtained from Sigma Aldrich. All solutions were prepared with deionized water of resistivity of $18.2 \mathrm{M} \Omega \mathrm{cm}$ or with phosphate buffered saline (PBS) solutions.

\subsection{Synthesis of molecularly imprinted polymers}

The synthesis protocol was optimized by varying the ratios of monomers, target and cross-linker (Supplementary Information Table S1) which is part of a bigger, previously conducted study on 2-MXP bulk MIPS. ${ }^{30}$ The best results were obtained by dissolving a mixture of the functional monomer methacrylic acid (MAA $1.02 \mathrm{mmol}$ ), crosslinker molecule ethylene glycol dimethacrylate (EGDMA 1.7 mmol), and initiator azobisisobutyronitril (AIBN $0.30 \mathrm{mmol}$ ) in dimethyl sulfoxide (DMSO) together with the template molecule $(0.17 \mathrm{mmol})$. The mixture was purged with $\mathrm{N}_{2}$ before the initiation of the polymerization. Polymerization was performed by heating the mixture up to $65^{\circ} \mathrm{C}$ for $12 \mathrm{~h}$, allowing for full completion of the reaction. MIPs and NIPs were milled seven times using a Fritsch Planetary Micro Mill Pulverisette 7 premium line (700 rpm, 5 minutes, $10 \mathrm{~mm}$ balls). After milling, the particles were sieved at $1.0 \mathrm{~mm}$ amplitude using a Fritsch Analysette 3 for 4 hours or until sufficient amount of polymer was on the collection plate to achieve microparticles with sizes smaller than $100 \mu \mathrm{m}$. Finally, the template molecule was removed from the MIP powders by continuous Soxhlet extraction with a 1:10 mixture of acetic acid and methanol for $12 \mathrm{~h}$, followed by further extraction with pure methanol for a further $12 \mathrm{~h}$. Extraction was verified using FTIR (see Supplementary Information Fig. S1). The MIP powder was then dried at $60^{\circ} \mathrm{C}$ overnight, yielding the extracted dried MIP powder. The NIP was prepared in the same manner, without the presence of the template molecule. MIP morphology was studied using scanning electron microscopy (see Supplementary Information Fig. S2).

\subsection{Batch rebinding}


Optical batch rebinding experiments were evaluated with a Shimadzu UV-3600 spectrophotometer. A 1 $\mathrm{mM}$ stock solution consisting of PBS and target substrate was used to prepare an array of solutions varying between $0-0.7 \mathrm{mM}$. To $5 \mathrm{ml}$ of each solution $20 \mathrm{mg}$ of MIP/NIP powder was added, and the resulting suspensions placed on an orbital shaker $(125 \mathrm{rpm})$ for $1 \mathrm{~h}$ at room temperature. After filtration, the free concentration of substrate in the filtrate was determined by UV-vis spectroscopy and HPLC analysis, allowing binding isotherms to be constructed. As a measure of specificity and in order to compare the different MIP compositions, the imprint factor (IF) was determined at $\mathrm{C}_{\mathrm{f}}=0.05 \mathrm{mM}$. To determine whether the MIP could selectively extract the target molecule from mixtures, selectivity tests were performed with other substrates. This was done for all MIPs under study.

\subsection{Preparation of dye-loaded MIP}

To $20 \mathrm{ml}$ of aqueous dye molecule $(1 \mathrm{mM}), 500 \mathrm{mg}$ of previously prepared MIP powder was added, and proceeded to be left on an orbital shaker $(250 \mathrm{rpm})$ for $2 \mathrm{~h}$. The resulting suspension was then filtered and the remaining filtered solid washed with distilled water until the filtrate ran colourless. The coloured MIP powder was then placed in an oven overnight at $90^{\circ} \mathrm{C}$ to yield a dry powder. 2-MXP MIPs were loaded with crystal violet, malachite green, methyl orange, basic blue, phenol red, or pararosailine respectively to study the SDC principle and to examine the effect of combining different dyes with different MIPs.

\subsection{Kinetic analysis of Substrate Displacement Colorimetry (SDC)}

The SDC assay was initiated by adding $20 \mathrm{mg}$ of dye-loaded MIP powder to $5 \mathrm{~mL}$ of aqueous 2-MXP (1 $\mathrm{mM})$. After varying fixed time intervals $(1,2,3,4,5,10,15$ and 20 minutes) of incubation on an orbital shaker, the solutions were filtered and the colour of the filtrate observed. If there was a significant amount of binding between the 2-MXP and the dye-loaded MIP, the dye would be displaced and released into solution, resulting in a colour change after filtration. Filtrates were examined both visually and optically using a Shimadzu UV-3600 spectrophotometer, to build a relationship between the dye displacement and the incubation time. 


\subsection{Substrate displacement Colorimetry (SDC) selectivity analysis}

The SDC assay was initiated by adding $20 \mathrm{mg}$ of dye-loaded MIP powder to $5 \mathrm{~mL}$ of aqueous analyte (1 $\mathrm{mM}$ ). After one minute of incubation on a shaker, the solutions were filtered and the colour of the filtrate observed. This was repeated for all MIPs under study and used to analyse samples containing caffeine, paracetamol, aspirin, and sucrose. If there was a significant amount of binding between the substrate and the dye-loaded MIP, the dye would be displaced and released into solution, resulting in a colour change after filtration. Filtrates were examined both visually and optically using a Shimadzu UV-3600 spectrophotometer. As NPS have ever evolving minor structural changes, the dye-loaded MIP was also exposed to other diarylethylamines, assessing if the assay would be able to cope with continuously changing compositions of NPS.

\subsection{SDC Quantitative analysis of loaded 2-MXP MIP}

To examine the potential for quantifying drugs aqueous solutions containing varying concentrations $(0.01$ - $1 \mathrm{mM}$ ) of 2-MXP and caffeine were incubated with $20 \mathrm{mg}$ of malachite green-loaded 2-MXP MIPs and shook on an orbital shaker $(125 \mathrm{rpm})$ for 5 minutes. The solutions were then filtered and the absorbance of the malachite green displaced into solution was determined by UV-vis spectroscopy. All measurements were performed in triplet and a mean absorbance and standard error was calculated for each concentration. The data were fit using Origin Pro 8.

\subsection{Proof-of-application: low-cost drug detection kit}

To examine the potential of the assay to rapidly detect the presence of 2-MXP in unidentified powders by means of a fast, low-cost direct test, PTFE syringe filters $(0.45 \mu \mathrm{m}$ pore size $)$ were loaded with dyecontaining MIP particles. To this extent, $20 \mathrm{mg}$ of malachite-green loaded MIP powder was suspended in $5 \mathrm{~mL}$ of distilled water after which the MIP solution was pushed through the filter to immobilize the MIp particles directly in the filter membrane. The filter proceeded to left to stand vertically for 30 minutes allowing any remaining distilled water to gravimetrically pass through, leaving the dried SDC MIP inside 
the filter. The filter proceeded to left to stand vertically for 30 minutes allowing any remaining distilled water to gravimetrically pass through, leaving the dried SDC MIP inside the filter. To assess qualitative functioning of the system, $5 \mathrm{~mL}$ solutions of target and analogues $(4 \mathrm{mg} / \mathrm{mL}$ of caffeine, $2-\mathrm{MXP}$, sucrose, aspiring and paracetamol) were passed through the filters at a rate of $5 \mathrm{~mL} \mathrm{~min}^{-1}$. The filtrates were collected and analyzed using visual inspection and UV-VIS spectrometry. To investigate the sensor's performance in impure street samples, varying concentrations of 2-MXP were mixed with caffeine and analyzed using the same method. The resulting absorbance readings were compared to a calibration curve obtained with a pure sample of 2-MXP.

\section{Results and discussion}

\subsection{Batch rebinding analysis}

The specificity and binding capacity of MIPs optimized for the detection of 2-MXP were analysed by means of UV-vis spectroscopy. The results obtained in batch rebinding experiments with both functionalized MIPs and their non-imprinted reference (non-imprinted polymers or NIPs) were used to construct binding isotherms by plotting the amount of target bound per gram of polymer $\left(\mathrm{S}_{\mathrm{b}}\right)$ in function of the free concentration $\left(\mathrm{C}_{\mathrm{f}}\right)$ of target remaining in solution. In order to determine the imprinting factor (IF), the data, shown in Fig. 2, were fit using an allometric (2-parameter) fit that reflects the heterogeneous nature of the binding sites within the MIP particles. The IF was calculated as the relative amount of binding by the MIP in comparison to the NIP at $0.05 \mathrm{mM}$ using the allometric fit, demonstrating various IF values and binding capacities (Table 1). Of the MIP/NIPs tested, MIP-24 demonstrated the highest IF of 1.83 and a binding capacity of $159 \mu \mathrm{mol} \mathrm{g}{ }^{-1}$.

\subsection{Batch rebinding analysis: optimizing dye-MIP coupling}


Rational design of the SDC assay was simulated by a similar batch rebinding experiment as the one described in the previous chapter. 2-MXP MIPs were incubated with six common, readily available dyes with structures that favour the formation of hydrogen bonds (see Supplementary Information Fig. S3). The resulting binding isotherms show that the MIP has the highest affinity for malachite green. The highest degree of specific binding, which was expressed as the binding factor (BF), was also observed for malachite green. BF was calculated in an analogous way to the imprinting factor (IF) for the target but distinguishes from this classical term as the dyes were not imprinted. The resulting BFs and the extinction coefficients for each of the dyes were summarized in Table 2. Malachite green was chosen as a dye due to its superior binding factor and resulting positive effect on the selectivity of the assay. In addition, the use of malachite green as a loading dye for 2-MXP MIPs was evaluated due to its high extinction coefficient, which should increase the assay's sensitivity.

To demonstrate that malachite green was indeed the best available dye to be used in this assay, MIP particles were loaded with crystal violet, methyl orange, basic blue, phenol red, pararosaniline and malachite green and exposed to 2-MXP. The colours of the resulting filtrates were observed with the naked eye and the absorption was measured using the spectrophotometer (Table 3). Malachite green has the highest degree of displacement, having a visually observable coloured filtrate (Supplementary Information Fig. S4). The other dyes did not produce a noticeable difference in colour of filtrate, although a small amount of crystal violet could be detected with the spectrophotometer. These findings are in line with their low BFs, indicating that they will mostly occupy non-specific binding areas of the polymer and are easily removed during the washing step. The displacement is presumed to occur at the nanocavities where the 2-MXP has a higher affinity than the dye, displacing it out of the cavities into the surrounding solution. As the coloured MIP particles will be removed by means of a syringe filter the filtrate will only be coloured when the dye was displaced.

\subsection{Batch rebinding analysis: optimizing dye displacement}


The observed binding factor of the dye is only one parameter that must be considered when performing substrate displacement. If the MIP has a greater affinity for the dye than for the target, no displacement will occur, and therefore IF also plays a role in the efficacy of the displacement process. To demonstrate this concept, previously synthesized MIPs for 2-MXP were incubated with malachite green in a similar manner to the previous rebinding experiments. The binding isotherms generated from this allowed for the calculation of the binding factors of the malachite green towards each MIP (Table 4). The difference between the IF and $\mathrm{BF}$ is designated $\triangle \mathrm{BF}$ and gives an indication to the differences in specific binding of the MIP towards malachite green and 2-MXP. As $\triangle \mathrm{BF}$ becomes more positive, the likelihood of dye displacement increases. This is due to the target molecule having a higher affinity towards the MIP than the currently bound dye molecule, as the imprinting effect favours the target molecule. From the experimental data a curve was constructed, depicting this mathematical relationship between BF and IF (Fig. 3). The dashed line in the graph represents the point where BF and IF are equal. Any MIPs that have data points below this line will not exhibit displacement properties as the binding of the dye is greater than that of 2MXP. Confirmation of this theory was conducted by preloading each of the MIPs with malachite green, and then incubating the loaded MIPs with 2-MXP $(1 \mathrm{mM})$. MIP-24, the MIP with the highest $\Delta \mathrm{BF}$, shows clear displacement of the dye that can be visually observed (Supplementary Information Fig. S5) while MIP 25 displaces less dye making visual observation more difficult. These results were confirmed with UV-vis spectroscopy (Table 4).

\subsection{SDC: Incubation time dependency}

To get an insight into the kinetic behaviour of the dye displacement process, the effect of varying the incubation time on dye displacement was released. The malachite green-loaded MIPs were incubated with 2-MXP (1 mM) for different amounts of time, varying from 1 minute to 20 minutes. The UV absorbance of the dye displaced was measured for each time period, to determine the optimal operating time (Fig 4). A clear coloured filtrate is already observed within a minute after incubation and a time-dependent, linear increase in colour intensity could be observed during the first 10 minutes. The UV-vis data indicate that the 
curve levels after 10 minutes eventually plateauing at 20 minutes, indicating that no additional dye is being released from the MIP. These results indicate that detecting trace amounts of narcotics in unknown powders in a quantitative manner will probably require longer time intervals but, depending on the concentration of drugs in the sample, a fast test can also be performed.

\subsection{Selectivity of the SDC}

The selectivity of the MIP towards other pharmaceutical compounds (paracetamol and aspirin) and common adulterants (caffeine and sucrose) was assessed to come to a primary evaluation of the MIP's potential to discriminate an NPS from other morphologically similar white powders. The batch rebinding analysis, (shown in Supplementary Information Fig. S6) indicates that the MIP is very selective towards its target. The binding capacity of the MIP for all these compounds (structures summarized in Supplementary Information Fig. S7) is lower in comparison to the template and IFs do not significantly deviate from 1.The assay shows a similar trend; incubating MIPs, pre-loaded with malachite green, with any of the analogue powders, did not result in any visible colour change in the filtrate (See Supplementary Fig. S8). Further analysis of the filtrates by means of UV-absorbance readings ( $\lambda=616 \mathrm{~nm}$, Malachite green's most intense absorbance peak, Table 5) confirmed that the dye was not present in the filtrate. The presence of 2-MXP, is the only compound tested that elicited a response from the dye loaded MIP, showing the selectivity of the MIP against other possible chemical architectures.

However, 2-MXP falls in the class of compounds known as diarylethylamines that are known to induce psychoactive effects when consumed. Drug producers tend to slightly modify the structure of these compounds to avoid legislation. Therefore, known psychoactive diarylethylamines are shown to be structural analogues or region-isomers of 2-MXP (structures summarized in Supplementary Information Fig. S9), giving them a high affinity towards the MIP. This apparent limited selectivity of the MIP system could be of great analytical use because it allows for the detection of other diarylethylamines without the need for synthesizing a MIP for each new NPS of this class that reaches the market. MIP particles, preloaded with malachite green, were incubated with other aqueous solutions of diarylethylamines, leading to 
displacement of the dye and visibly coloured filtrates (Supplementary Information Fig. S10), UVabsorbance readings were taken to confirm displacement (Table 5). This illustrates that the assay can be tailored towards a broader array of analytes sharing a similar structure.

\subsection{SDC: quantitative analysis}

To verify whether it is possible to quantify the colorimetric response, malachite green-loaded and crystal violet-loaded MIP powders were incubated with increasing amounts of 2-MXP and caffeine $(0.01-1 \mathrm{mM}$ for both compounds) and analyzed using a UV-vis spectrometer to determine the amount of target present in the analyte. The data shown in Figure 5a shows that the MIP releases dye in a concentration-dependent manner when exposed to increasing amounts of 2-MXP. Exposing the dye-loaded MIP to caffeine does not result in any measurable displacement of the dye over the entire concentration range. In order to determine the limit-of-detection (LoD) the lower concentration range was plotted and analyzed separately in Figure $5 b$. The data were fit using a linear regression line $\left(\mathrm{R}^{2}=0.95653\right)$ and the $3 \sigma$ method was used to establish a detection limit of $50 \mu \mathrm{M}$. This indicates that the technology can be used to detect micrograms of unknown powders dissolved in milliliter amounts of water.

\subsection{Low-cost drug identification kit}

Loading of the PTFE filters with dye-containing MIP particles leads to intense coloring of the syringe filter providing optical verification that immobilization was successful (see Supplementary Figure S11). Pushing a solution containing 2-MXP through the syringe filter will lead to rebinding of the target to the MIP and subsequent displacement of the dye into the filtrate. Reference experiments using solutions containing common adulterants or legal pharmacological compounds does not result in a color change of the filtrate (Supplementary Figure S11). The experiment was repeated with a dilution series of 2-MXP to create a calibration curve. Repeating the experiment with powders consisting of a mixture of 2-MXP and a common adulterant (caffeine) illustrates that the low-cost kit is able to faithfully detect the target in an unknown powders at concentrations down to 1 percent (Figure 6). 


\section{Conclusion}

The results in this paper nicely illustrate the underlying principles of a substrate displacement strategy that relies upon MIPs for their selectivity. Structural similarities and similar spatial orientation of functionalities between the dye and target molecule (2-MXP) were exploited to create a selective detection assay. The assay also allows for the detection of trace amounts of drugs which can be attributed to the high extinction coefficient of malachite green. Although the assay has demonstrated to be able to selectively discriminate between NPS and compounds that can yield false negatives, it is not too selective to enable drug producers and users to evade the test by slightly modifying their synthesis process as the test is responsive to other diarylethylamines as well. The fast test is able to detect 2-MXP in mixed powders in concentrations as low as $0.015 \mathrm{mg} \mathrm{mL}^{-1}$. The current assay has great potential for low -cost, routine screening of unknown powders for the presence of narcotics, but the detection of drugs or metabolites in biological samples would require lower detection ranges $[31,32]$. However, most of this can be attributed to the limited specificity of the MIP that can be improved in the future by using more advanced imprinting strategies such as the double molecular imprinting approach or by using e.g. solid phase extraction to create nano-sized MIPs that react faster and more accurate to the presence of their target $[33,34]$.

\section{Acknowledgements}

The authors are grateful for funding through the "Limburg Meet" project funded by the province of Limburg, the Netherlands and the Royal Society of Chemistry (Research Fund RF18-2881 and RSC mobility grant). Technical support by numerous Maastricht Science Programme undergrad students is greatly appreciated.

\section{Author Contributions Statement}


All MIPs were synthesized by J.L. and input on the synthesis and template removal protocol was provided by M.P., H.D. and T.J.C. Colorimetric experiments were performed by J.L. in close cooperation with R.R. and B.H. Data fitting and processing was done by B.v.G., K.E. and J.L. Input on drug assay development was provided by E.S.R. and K.E. The manuscript was jointly written by J.L. and K.E.

\section{Appendix A. Supplementary data}

Additional data is available as Supporting Information to this article

\section{References}

1. Wulff, G. Enzyme-like Catalysis by molecularly imprinted polymers. Chem. Rev. 102, 1-28 (2002).

2. Sellergen, B. \& Allender, C.J. Molecularly imprinted polymers: a bridge to advanced drug delivery. Adv. Drug Del. Rev. 57, 1733-1741 (2005).

3. Whitcombe, M.J., Kirsch, N. \& Nicholls, I.A. Molecular imprinting science and technology: a survey of the literature for the years 2004-2011. J. Mol. Recogn. 27, 297-401 (2014).

4. Chianella, I. et al. Direct replacement of antibodies with molecularly imprinted polymer nanoparticles in ELISA — development of a novel assay for vancomycin. Anal. Chem. 85, 8462-846 (2013).

5. Yano, K.; \& Karube, I. Molecularly Imprinted Polymers for Biosensor Applications. Trends Anal. Chem. 18, 199-204 (1999).

6. Haupt, K. \& Mosbach, K. Molecularly imprinted polymers and their use in biomimetic sensors. Chem. Rev. 100, 2495-2504 (2000).

7. Ye, L. \& Haupt, K. Molecularly imprinted polymers as antibody and receptor mimics for assays, sensors and drug discovery. Anal. Bioanal. Chem. 378, 1887-1897 (2004).

8. Wackers, G et al. Array formatting of the heat-yransfer method (HTM) for the detection of small organic molecules by molecularly imprinted polymers. Sensors 14, 11016-11030 (2014).

9. King, H.A. et al. Generation of ribosome imprinted polymers for sensitive detection of translational responses. Sci. Rep. 7, 6542 (2017). 
10. Steen Redeker, E. et al. Biomimetic bacterial identification platform based on thermal wave transport analysis (TWTA) through surface-imprinted polymers. ACS Inf. Dis. 3, 388-397 (2017).

11. Selvoni, G. \& Marrazza, G.; MIP-based sensors: promising new tools for cancer biomarker determination. Sensors 17, 718 (2017).

12. Vandenryt, T et al. Single-shot detection of neurotransmitters in whole-blood samples by means of the heat-transfer method in combination with synthetic receptors. Sensors 17, 2701 (2017).

13. Fernandes, R., Dinc, M., Raimundo Jr., I. \& Mizaikoff, B. Molecularly imprinted core-shell hybrid microspheres for the selective extraction of vanillin. Analytical Methods 9, 2883-2889 (2017).

14. Diliën, H.; et al. Label-free detection of small organic molecules by molecularly imprinted polymer functionalized thermocouples: toward in vivo applications. ACS Sens. 2, 583-589 (2017).

15. Zhao, W. et al. Molecularly imprinted polymeric nanoparticles decorated with Au NPs for highly sensitive and selective glucose detection. Biosens. Bioelectron. 100, 497-503 (2018).

16. Canfarotta, F. et al. A novel thermal detection method based on molecularly imprinted nanoparticles as recognition elements. Nanoscale 10, 2081-2089 (2018).

17. Vlatakis, G., Andersson, L.I., Muller, R. \& Mosbach, K. Drug assay using antibody mimics made by molecular imprinting. Nature 361, 645-647 (1993).

18. Haupt, K., Dzgoev, A. \& Mosbach, K. Assay system for the herbicide 2,4-dichlorophenoxyacetic acid using a molecularly imprinted polymer as an artificial recognition element. Anal Chem. 70, 628-631 (1998).

19. Urraca, J.L., Moreno-Bondi, M.C., Orellana, G., Sellergren, B. \& Hall, A.J. Molecularly imprinted polymers as antibody mimics in automated on-line fluorescent competitive assays. Anal Chem. 79, 4915-4923 (2017).

20. Shi, C., Liu, X.Y., Song, L.Y., Qiao, X.G. \& Xu, Z.X. Biomimetic enzyme-linked immunosorbent assay using a hydrophilic molecularly imprinted membrane for recognition and fast determination of trichlorfon and acephate residues in vegetables. Food Anal Meth. 8, 2496-2503 (2015). 
21. Koh, K.N., Araki, K., Ikeda, A., Otsuka, H. \& Shinkai, S. Reinvestigation of Calixarene-Based Artificial-Signaling Acetylcholine Receptors Useful in Neutral Aqueous (Water/Methanol) Solution. $J$. Am. Chem. Soc. 118,755-758 (1996).

22. Tan, J., Li, R. \& Jiang, Z. T. Discrimination of fresh fruit juices by a fluorescent sensor array for carboxylic acids based on molecularly imprinted titania. Food Chem. 165, 35-41 (2014).

23. Greene, N.T. \& Shimizu, K.D. Colorimetric molecularly imprinted polymer sensor array using dye displacement. J. Am. Chem. Soc. 127, 5695-5700 (2005).

24. Lee, J.D., Greene, N.T., Rushton, G.T., Shimizu, K.D. \& Hong, J.I. Carbohydrate Recognition by Porphyrin-Based Molecularly Imprinted Polymers. Org. Lett. 7, 963-966 (2007).

25. Silverio, O.V., So, R.C., Elnar, K.J.S., Malapit, C.A., Cristine, M. \& Nepomuceno, M. Development of dieldrin, endosulfan, and hexachlorobenzene-imprinted polymers for dye-displacement array sensing. J. Appl. Pol. Sci. 14, 44401-44411 (2016).

26. Philip, M. \& Fu, S. A review of chemical 'spot' tests: A presumptive illicit drug identification technique. Drug Test Anal. 10, 95-108 (2018).

27. Buchanan, J.F. \& Brown C.R. 'Designer drugs'. A problem in clinical toxicology. Med Toxicol Adverse Drug Exp. 3, 1-17 (1998).

28. Bey, T. \& Patel, A. Phencyclidine intoxication and adverse effects: a clinical and pharmacological review of an illicit drug. Cal. J. Emerg. Med. 8, 9-14 (2007).

29. Castaneto, M.S. et al. Synthetic cannabinoids: epidemiology, pharmacodynamics, and clinical implications. Drug Alcohol Depend. 1, 12-41 (2014).

30. Lowdon, J.W. et al. Engineering molecularly imprinted polymers (MIPs) for the selective extraction and quantification of the novel psychoactive substance (NPS) methoxphenidine and its regioisomers. Analyst, 143, 2002 (2018).

31. Kunsman, G.W., Levine, B., Constantino, A. \& Smith, M.L. Phencyclidine Blood Concentrations in DRE Cases. J Anal Toxicol. 21, 498-502 (1997). 
32. Helander, A., Beck, O. \& Bäckberg, M. Intoxications by the dissociative new psychoactive substances diphenidine and methoxphenidine. Clin. Toxicol. 53, 446-453 (2015).

33. Dickert, F.L., Achatz, P., Halikias, K. Double molecular imprinting--a new sensor concept for improving selectivity in the detection of polycyclic aromatic hydrocarbons (PAHs) in water. Frensius J. Anal. Chem. 371, 11-15 (2001).

34. Canfarotta, F., Poma, A., Guerreiro, A., Piletsky, S. Solid-phase synthesis of molecularly imprinted nanoparticles. Nat. Protoc. 11, 443-455 (2016).

\section{Figures and Tables}

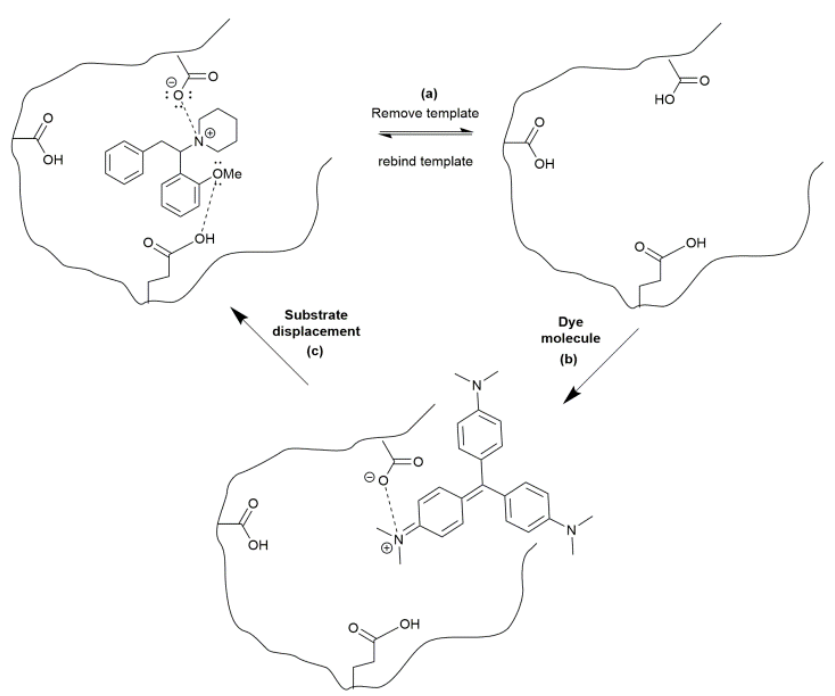

Fig. 1. Substrate displacement colorimetry principle. Extracted MIPs (2-MXP) are loaded with a structural similar dye (crystal violet). When the MIP is incubated with its original template, the dye is displaced from the binding pockets and released into the surrounding medium. 


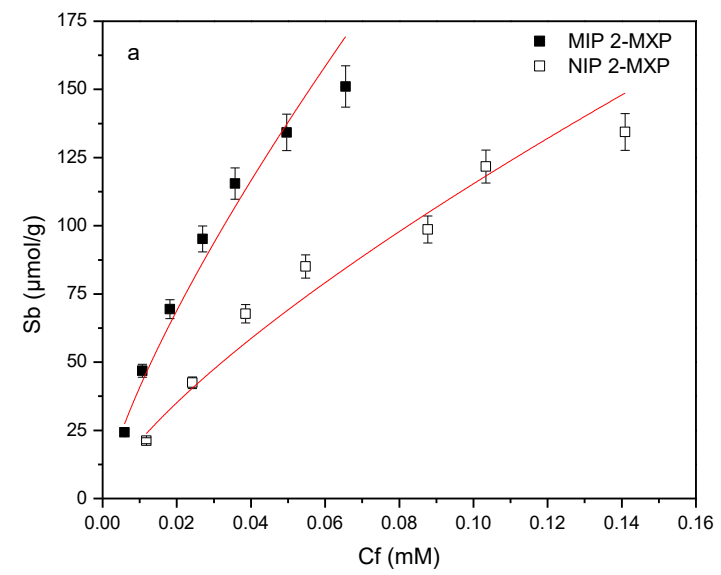

Fig. 2. Batch rebinding analysis on a UV-vis spectrometer: both a MIP imprinted for 2-MXP and a NIP reference were exposed to increasing concentrations of 2-MXP in water. Error bars are representations of the standard error over three measurements. The binding isotherms show that the MIP is able to bind the target in a specific manner and an imprinting factor of 1.83 can be observed at $0.05 \mathrm{mM}$.

Table 1. Imprint factors and binding capacities of the MIPs synthesized templated with 2-MXP

\begin{tabular}{c|cc} 
MIP & IF & Binding capacity $\left(\boldsymbol{\mu m o l ~ \mathbf { ~ g } ^ { - 1 } )}\right.$ \\
\hline MIP-21 & 1.02 & 141 \\
MIP-22 & 1.32 & 146 \\
MIP-23 & 1.01 & 156 \\
MIP-24 & 1.83 & 159 \\
MIP-25 & 1.07 & 149
\end{tabular}

Table 2. Binding factors and extinction coefficients for methyl orange, crystal violet, phenol red, basic blue, pararosaniline and malachite green upon incubation with MIP-24 particles.

\begin{tabular}{c|cc} 
Dye & Binding Factor $\mathbf{( B F )}$ & $\begin{array}{c}\text { Extinction coefficient } \\
\left(\mathbf{x 1 0}^{\mathbf{3}} \mathbf{M}^{\mathbf{- 1}} \mathbf{c m}^{\mathbf{- 1}} \mathbf{)}\right.\end{array}$ \\
\hline Malachite green $(\lambda=616 \mathrm{~nm})$ & 1.19 & 148,000 \\
Crystal Violet $(\lambda=590 \mathrm{~nm})$ & 1.05 & 87,000 \\
Methyl Orange $(\lambda=464 \mathrm{~nm})$ & 0.39 & 20,500 \\
Basic Blue $(\lambda=616 \mathrm{~nm})$ & 0.81 & 62,000 \\
Pararosaline $(\lambda=616 \mathrm{~nm})$ & 0.99 & 80,000 \\
Phenol Red $(\lambda=432 \mathrm{~nm})$ & 0.98 & 55,000
\end{tabular}


Table 3. Absorbance of the filtrates collected when MIP-24 loaded with either malachite green, phenol red, crystal violet, methyl orange, pararosaniline, or basic blue, was exposed to 2-MXP (1 mM) for 1 minute.

\begin{tabular}{c|cc} 
Pre-loaded Dye & Absorbance & Colour of filtrate \\
\hline Malachite green $(\lambda=616 \mathrm{~nm})$ & 0.0750 & Blue \\
Crystal Violet $(\lambda=590 \mathrm{~nm})$ & 0.0182 & Colourless \\
Methyl Orange $(\lambda=464 \mathrm{~nm})$ & 0.0000 & Colourless \\
Basic Blue $(\lambda=616 \mathrm{~nm})$ & 0.0000 & Colourless \\
Pararosaline $(\lambda=616 \mathrm{~nm})$ & 0.0000 & Colourless \\
Phenol Red $(\lambda=432 \mathrm{~nm})$ & 0.0000 & Colourless
\end{tabular}

Table 4. Imprint factors and binding factors when MIPs have been exposed to the target 2-MXP, and the dye malachite green, $\Delta \boldsymbol{B} \boldsymbol{F}$ indicates the difference in binding between the target molecule and the dye. Absorbance readings displayed relate to the absorbance of the malachite green after the dye loaded MIP has been incubated with 2-MXP $(1 \mathrm{mM})$, and the colour of the filtrate observed.

\begin{tabular}{c|ccccc} 
MIP & IF (2-MXP) & $\begin{array}{c}\text { BF (Malachite } \\
\text { green) }\end{array}$ & $\Delta \boldsymbol{B} \boldsymbol{F}$ & $\begin{array}{c}\text { Absorbance } \\
(\boldsymbol{\lambda}=\mathbf{6 1 6} \mathbf{n m})\end{array}$ & $\begin{array}{c}\text { Colour of } \\
\text { filtrate }\end{array}$ \\
\hline MIP-21 & 1.02 & 1.42 & -0.40 & 0.0000 & Colourless \\
MIP-22 & 1.32 & 2.33 & -1.01 & 0.0000 & Colourless \\
MIP-23 & 1.01 & 1.22 & -0.21 & 0.0000 & Colourless \\
MIP-24 & 1.83 & 1.19 & 0.64 & 0.0750 & Blue \\
MIP-25 & 1.07 & 1.04 & 0.03 & 0.0121 & colourless
\end{tabular}




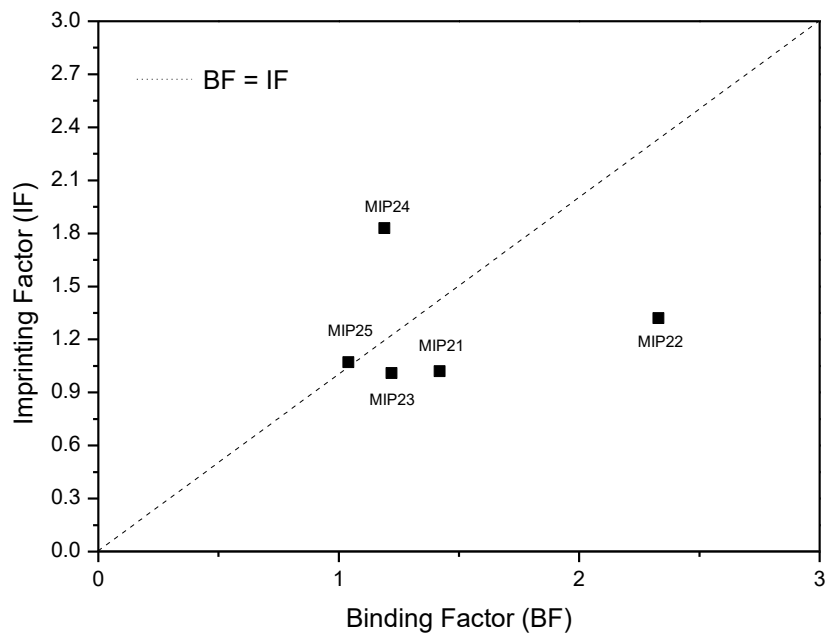

Fig 3. Imprint factor - Binding factor graph for varying compositions of MIPs that are imprinted for 2MXP. Imprint factor is in relation to 2-MXP, and binding factor is in relation to malachite green.

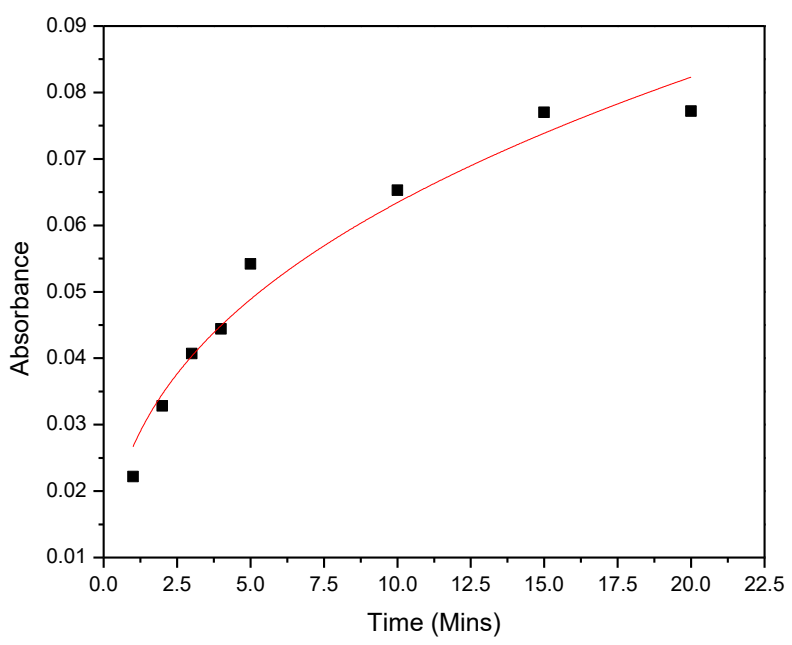

Fig 4. Time dependent elution isotherm for when malachite green loaded MIP-24 is incubated with 2-MXP (1 mM) for varying time intervals. The UV absorbance of the malachite green was measured using a UVspectrometer, to determine the amount of dye displaced.

Table 5. Compounds of different chemical classes (a) and compounds of the same class as 2-MXP (b) were incubated with the malachite green loaded MIP. The filtrates were then collected UV-absorbance measured $(\lambda=616 \mathrm{~nm})$ and colour of filtrate observed. 


\begin{tabular}{c|cc} 
(a) Different substrate class & Absorbance & Colour \\
\hline Blank & 0 & Colourless \\
Caffeine & 0 & Colourless \\
2-MXP & 0.0750 & Blue \\
Paracetamol & 0 & Colourless \\
Sucrose & 0 & Colourless \\
Aspirin & 0 & Colourless \\
\hline (b) Same substrate class & Absorbance & Colour \\
\hline 2-MXP & 0.0750 & Blue \\
3-MXP & 0.0217 & Blue \\
4-MXP & 0.0261 & Blue \\
Diphenidine & 0.0193 & Blue \\
2-MEPE & 0.0131 & Blue \\
3-MEPE & 0.0103 & Blue \\
4-MEPE & 0.0163 & Blue \\
Ephenidine & 0.0998 & Blue
\end{tabular}
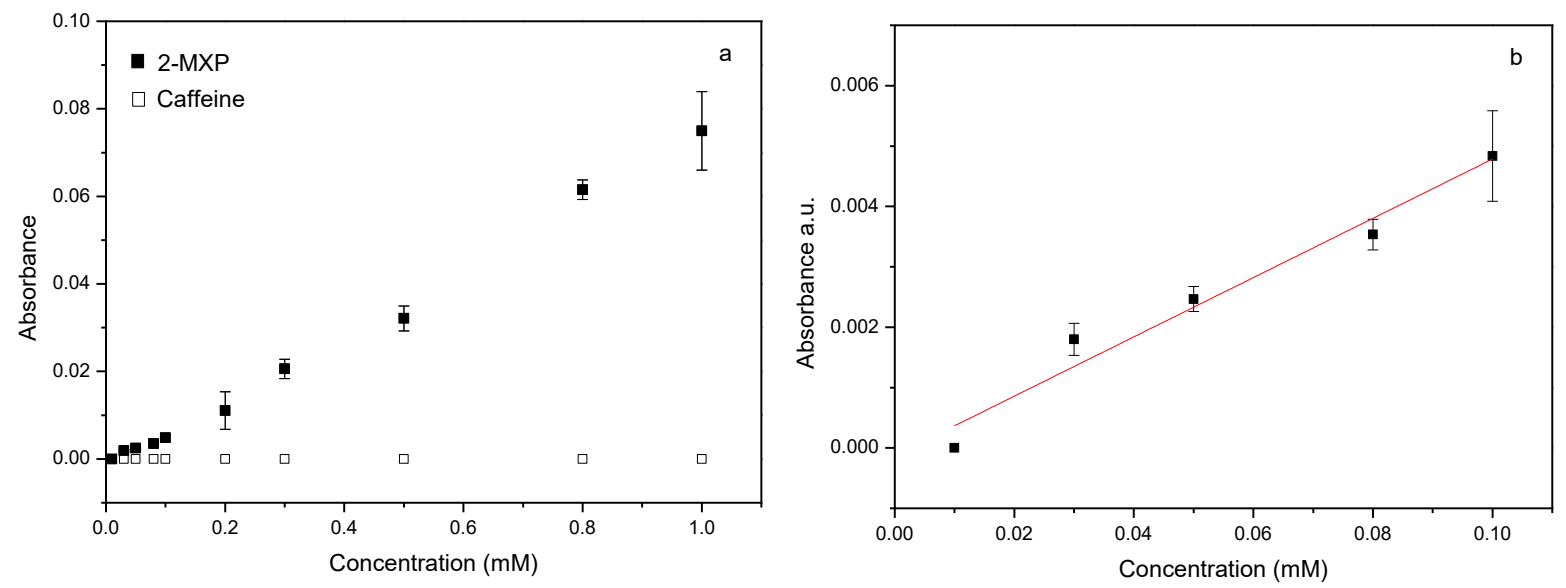

Fig. 5. Quantitative 2-MXP assay: to determine the limit-of-detection (LoD) for the SDC assay using 2MXP MIPs loaded with malachite green. The plot over a wide concentration range indicates that the response for 2-MXP can be quantified while exposing the MIP to an increasing concentration of caffeine does not result in any measurable displacement of the dye (a). Zooming in on the lower concentration range shows a linear increase $(\mathrm{R} 2=0.95653)$ and an $\mathrm{LoD}$ of $50 \mu \mathrm{M}$ was calculated using the $3 \sigma$ method $(\mathrm{b})$. 


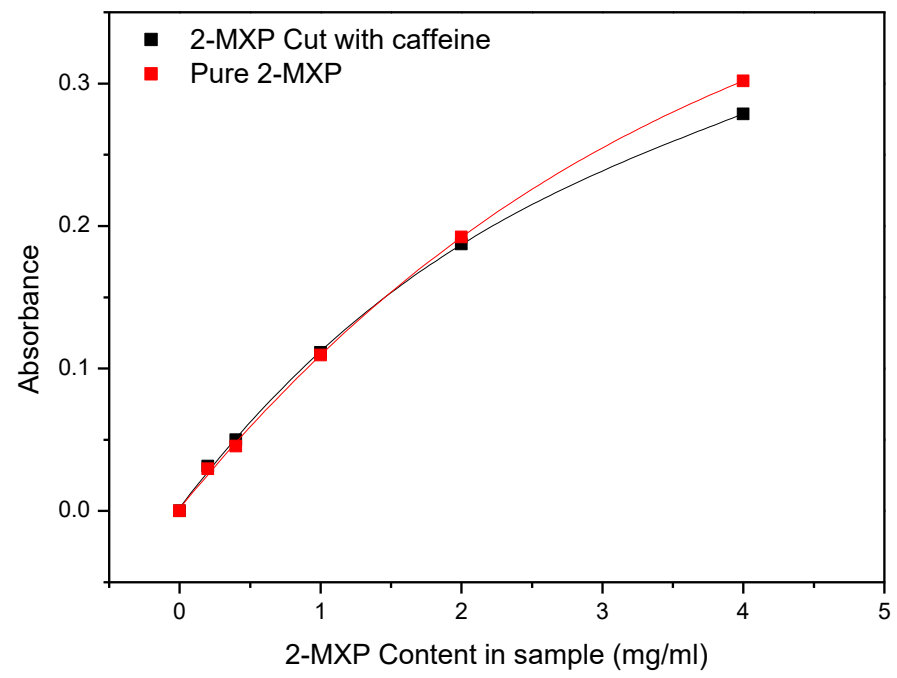

Fig. 6. Proof-of-application of a low-cost drug test kit. Dye-loaded MIP particles were immobilized into syringe filters. Solutions containing both the pure compound as well as a mixture consisting of the target and a common adulterant were passed through the filter and the absorption of the filtrate was measured. The results indicate that the tests is able to detect the target in a powder mixture.

\section{Vitae}

Mr. Joseph W. Lowdon MChem (Hons) obtained an integrated master's degree in chemistry from Manchester Metropolitan University in 2017. Over the course of his studies, he participated in multiple internships in the fields of synthesis and characterization of New Psychoactive Substances, synthesis of fluorescent monomers, and intelligent design of molecularly imprinted polymers. Since November 2017 he has begun to obtain his $\mathrm{PhD}$ in chemical and biological biosensing within the Smart Devices Research Unit of the Faculty of Science and Engineering at Maastricht University. His research interests include synthesis of biomimetic polymers for bio- and chemical sensing, biocompatible materials for drug delivery and the implemeAntation of new technologies for healthcare management.

Dr. Kasper Eersels obtained a master's degree in bioelectronics and nanotechnology and a PhD in science at Hasselt University in 2009 and 2014 respectively. He continued working in the BIOSensor group at Hasselt University until 2015 as a postdoc after which he moved to KULeuven to work as a postdoctoral 
fellow at KULeuven in the Soft Matter and Biophysics section of the department of Physics and Astronomy. Since 2017 he is employed as a postdoc within the Smart Devices Research Unit of the Faculty of Science and Engineering at Maastricht University. His research interests include bio- and chemosensor development, biomedical application engineering and implementation of technology into healthcare.

Mr. Renato Rogosic MSc. obtained a degree in Biomechanics and Biomaterials from Politecnico di Milano in 2017. During his studies, he focused on the application of engineering in various aspects of biomedical sciences. He participated in different internships in material surface modification for biological applications and microfluidic design for cell culture. Since November 2017, he has begun to obtain his $\mathrm{PhD}$ in biosensors within the Smart Devices Research Unit of the Faculty of Science and Engineering at Maastricht University. His research interests include design and fabrication of microfluidic devices for bio sensing applications and development of new sensing techniques based on innovative technologies.

Mr. Benjamin Heidt M.Sc. obtained a bachelor's degree in Biochemistry at the University of Applied Sciences Mannheim followed by a master's degree in Applied Life Sciences (Bio-, Pharma- and Medical Sciences) at the University of Applied Sciences Kaiserslautern in 2017. During his studies he took part in a range of research projects focused around biosensing, including microarray bacteria screening, bioreactor glucose monitoring and cell-integrated redox sensors. In January 2018 he has begun to obtain his PhD in chemical and biological biosensing within the Smart Devices Research Unit of the Faculty of Science and Engineering at Maastricht University. His research interests include functional surfaces for selective detection of pathogens as well as biosensor development and their implementation in healthcare settings.

Dr. Hanne Diliën obtained a master's degree in chemistry (molecular design and synthesis) at KULeuven in 2006. She started a $\mathrm{PhD}$ project in the development of low band gap polymers at Hasselt University in cooperation with IMEC and obtained her $\mathrm{PhD}$ in sciences in 2011. As a postdoctoral fellow she continued working at Hasselt University as a member of the Nanobiotechnology and Bioelectronics group. Since 2013 she is an assistant professor in the Maastricht Science Programme at Maastricht University. Furthermore she is part of the Smart Devices research group, mainly focusing on the development of biosensors. Dr. 
Diliën's research interests are focused on organic and polymer synthesis for applications in the (bio)medical field; such as synthesis of pharmaceuticals, hydrogels,...

Erik Steen Redeker obtained his Master's degree in chemistry, specialising in biochemistry, at the Utrecht University and his Ph.D. in Sciences at the Antwerp University. In 2008 he was appointed as a postdoctoral fellow at the Hasselt University where he co-founded the Biomolecule Design Group. He is currently appointed at the Maastricht University as a lecturer and member of the Smart Devices research group. His research interests are focused on the rational (re)design, development and (bio)synthesis of biopolymers and their integration into biosensors. He uses synthetic biology approaches and other techniques inspired by and derived from strategies found in nature.

Dr. Marloes Peeters obtained a master's degree in chemistry and chemical engineering from Eindhoven University of Technology of Technology. Subsequently, she moved to Hasselt University to complete a $\mathrm{PhD}$ on biosensing using Molecularly Imprinted Polymers in 2013. This was followed by postdoctoral work at Hasselt University and at Queen Mary University of London. She started her independent research career at Manchester Metropolitan University in 2015, where she leads a group that works in the field of biosensors. In 2018, she was promoted to senior lecturer. Her research interests include the development of novel biosensor platforms that can be used for sustainable healthcare and environmental applications.

Dr. Bart van Grinsven received a master's degree in bioelectronics and nanotechnology from Hasselt University in 2007. Subsequently, he was employed by TNO as a development engineer before returning to the BIOSensor group of Hasselt University in 2008. After obtaining his $\mathrm{PhD}$ in 2012 and working as a postdoctoral researcher in the same group, he moved to Maastricht University in 2014. Currently, he holds the independent position of assistant professor within the research group of professor Thomas Cleij at Maastricht University, leading the biosensor research line. His field of interest includes biosensor development, PoC testing and biomedical device engineering. 
Prof. Dr. Thomas Cleij obtained his Ph.D. in 1999 at Utrecht University in polymeric materials science. Subsequently, he was appointed as assistant professor at Louisiana State University and associate and full professor at Hasselt University. In 2009, he became involved with the efforts to start natural science education at Maastricht University. This resulted in the Maastricht Science Programme, which he started in 2011 as Dean and Programme Director. He has been appointed at Maastricht University as a full professor since 2012 and Dean sciences since 2015. He is the head of the Nano(Bio)Technology and Bio-Electronics research group and his research interests are focused on the combination and interplay of novel functional polymeric materials and advanced applications in the life sciences. Examples of this research at the interface between chemistry, life science and physics include polymer based biosensors, molecularly imprinted polymers (MIPs) for bio-analytical applications and polymeric hydrogels for tissue engineering. 